\title{
Staphylococcal Adhesion and Host Cell Invasion: Fibronectin-Binding and Other Mechanisms
}

\author{
Jérôme Josse ${ }^{1}$, Frédéric Laurent ${ }^{1,2,3,4 *}$ and Alan Diot ${ }^{1}$ \\ ${ }^{1}$ International Center for Infectiology Research, INSERM U1111, CNRS UMR5308, ENS Lyon, Lyon 1 University, Lyon, \\ France, ${ }^{2}$ Institute for Infectious Agents, Hôpital de la Croix-Rousse, Hospices Civils de Lyon, Lyon, France, ${ }^{3}$ French National \\ Reference Centre for Staphylococci, Lyon, France, ${ }^{4}$ Microbiology-Mycology Department, Institut des Sciences \\ Pharmaceutiques et Biologiques de Lyon, Lyon, France
}

Opportunistic bacteria from the genus Staphylococcus can cause life-threatening infections such as pneumonia, endocarditis, bone and joint infections, and sepsis. This pathogenicity is closely related to their capacity to bind directly to the extracellular matrix or to host cells. Adhesion is indeed the first step in the formation of biofilm or the invasion of host cells, which protect the bacteria from the host immune system and facilitate chronic infection. Adhesion relies on the expression of a repertoire of surface proteins called adhesins, notably microbial surface components recognizing

OPEN ACCESS

Edited by:

Régine Talon,

INRA - Centre Auvergne

Rhône-Alpes, France

Reviewed by:

Dirk Linke,

University of Oslo, Norway

Michael Edward Konkel,

Washington State University,

United States

${ }^{*}$ Correspondence:

Frédéric Laurent

frederic.laurent@univ-lyon1.fr

Specialty section:

This article was submitted to Infectious Diseases,

a section of the journal

Frontiers in Microbiology

Received: 13 September 2017 Accepted: 23 November 2017 Published: 05 December 2017

Citation:

Josse J, Laurent F and Diot A (2017)

Staphylococcal Adhesion and Host Cell Invasion: Fibronectin-Binding

and Other Mechanisms.

Front. Microbiol. 8:2433.

doi: 10.3389/fmicb.2017.02433 adhesive matrix molecules. In this short review, we discuss the main pathway (FnBPFn- $\alpha 5 \beta 1$ integrin), as well as alternatives, through which Staphylococcus aureus adheres to and then invades non-professional phagocytic cells. We then examine the corresponding mechanisms for coagulase negative staphylococci. There is currently a little understanding of the molecular mechanisms that lead to internalization. Filling this gap in the literature would therefore be an important step toward limiting the duration of staphylococci infections in clinical practice.

Keywords: staphylococci, fibronectin-binding protein, adhesion, non-professional phagocytic cell, cell invasion

\section{INTRODUCTION}

Staphylococci are commensal bacteria that make up a large part of the microbiota of skin and mucous membranes. In pathogenic conditions, they cause opportunistic and life-threatening infections such as pneumonia, endocarditis, bone and joint infections, and sepsis (Lowy, 1998; Becker et al., 2014). So far, 47 species and 23 sub-species of staphylococci have been identified, of which a few are coagulase-positive, such as $S$. aureus or $S$. pseudintermedius, but most are coagulasenegative species (CoNS), e.g., S. epidermidis, S. lugdunensis, S. saprophyticus, or S. haemolyticus (Becker et al., 2014). Like most bacteria, staphylococci express a broad range of surface proteins involved in their adhesion to extracellular matrix (ECM), plasma proteins or directly to host cells. The most prevalent of these proteins are the microbial surface component recognizing adhesive matrix molecules (MSCRAMMs), also found in enterococci and streptococci (Patti et al., 1994). All MSCRAMMs share a similar structure, with two adjacent IgG-folded domains mediating their attachment to components of the host ECM such as collagen, fibrinogen, or Fn (Becker et al., 2014; Foster et al., 2014). This binding capacity is closely related to the pathogenicity of staphylococci since their adherence to ECM or plasma proteins is a crucial step in the formation of biofilm and in the invasion of host cells (Löffler et al., 2014; Moormeier and Bayles, 2017). 
In this review, we discuss the mechanisms of cell adherence and internalization of coagulase-positive and -negative staphylococci. The main focus will be on the role of the ECM protein Fn and staphylococcal Fn-binding proteins (FnBPs) in the adhesion to and invasion of non-professional phagocytic cells (NPPCs) such as epithelial cells, endothelial cells, fibroblasts, and osteoblasts.

\section{Staphylococcus aureus ADHESION AND INTERNALIZATION BY HOST CELLS}

\section{The FnBP-Fn- $\alpha 5 \beta 1$ Integrin Pathway}

The capacity of $S$. aureus to adhere to cells has been known since the early 1980s (Aly et al., 1980) and has been demonstrated for both primary cells and cell lines from various tissues (Ellington et al., 1999; Kerro Dego et al., 2002). Host cell adhesion mainly involves Fn forming a bridge between $\alpha 5 \beta 1$ integrin on the cellular side and Fn binding proteins (FnBPs, which are MSCRAMMs) on the bacteria (Tran Van Nhieu and Isberg, 1993; Sinha et al., 1999; Fowler et al., 2000; Grundmeier et al., 2004). This step is a prerequisite for any internalization into NPPCs; indeed, DU5883, an isogenic mutant of S. aureus NCTC 8325-4 defective in FnBP expression, cannot invade NPPCs (Fowler et al., 2000).

There are two FnBP isoforms in S. aureus, FnBPA and FnBPB, encoded respectively by the $f n b A$ and $f n b B$ loci, with very similar domain organizations and sequences (Jönsson et al., 1991; Burke et al., 2010). However, their presence varies across the population (Peacock et al., 2000). They consist of an amino-terminal secretion signal sequence followed by an A domain that is closely related to fibrinogen-binding protein clumping factor A (ClfA, Figure 1A). This domain can bind fibrinogen and elastin and is involved in Fn binding (Wann et al., 2000; Roche et al., 2004; Burke et al., 2011). The A domains of FnBPA and FnBPB only share $40 \%$ sequence identity (Burke et al., 2010). The A domain is followed by tandem repeats of Fn-binding regions (FnBRs, 95\% identity between FnBPA and FnBPB), 11 in FnBPA versus 10 in FnBPB (Figure 1A). This additional FnBR, along with the higher Fn affinity of certain FnBRs, might explain FnBPA's higher overall affinity for Fn and the fact that FnBA alone is sufficient for adhesion and cell invasion (Grundmeier et al., 2004; Testoni et al., 2011). Finally, the C-terminal peptidoglycan-binding motif (LPXTG) and the wall and membrane spanning domains anchor FnBPs to the cell wall.

In vivo, the deletion of either FnBP strongly attenuates the ability of strain SH1000 to colonize the kidney and multiply, and to cause fatal sepsis in mice. Although FnBPA contributes more to these symptoms than FnBPB does, both FnBPs appear necessary for the development of severe infections (Shinji et al., 2011). This observation suggests that cooperation between the two FnBPs may be required for strong cell adhesion or to efficiently trigger the internalization pathway in vivo.

The high affinity and specificity of FnBPs for Fn is conferred by the tandem $\beta$-zipper structure they form together (Fowler et al., 2000; Schwarz-Linek et al., 2003). Fn bridges S. aureus and the host cell through its binding to cellular $\alpha 5 \beta 1$ integrin (Fowler et al., 2003). The efficiency of this adhesion varies both between cell types - one high-affinity FnBR is sufficient for adhesion and invasion in endothelial cells (Edwards et al., 2010) but three are needed in keratinocytes (Edwards et al., 2011) - and S. aureus strains. Indeed, whereas studies have shown that isolates from infections of cardiovascular devices have amino acid changes in the FnBR domain that increase the affinity for Fn (Hos et al., 2015), this is not the case for isolates from prosthetic joint infections (Eichenberger et al., 2015). Likewise, certain methicillin-resistant $S$. aureus strains involved in endovascular infections harbor an additional FnBR that, together with a substitution in FnBR 11, reduces its affinity for Fn but promotes cell invasion. Although this fits with the hypothesis that the pathogenesis of endovascular infections involves the invasion of endothelial cells, it seems to go against the idea that better adhesion promotes internalization. However, it is estimated that one FnBP can bind 6-9 Fn molecules (Bingham et al., 2008) and thus could cluster $\alpha 5 \beta 1$ integrins on the cell surface to trigger the efficient intracellular signaling required for internalization. One hypothesis is that when affinity for Fn decreases, more interactions are needed to achieve sufficient cell adhesion, mobilizing more Fn and $\alpha 5 \beta 1$ integrins and thus leading to their clustering.

The signaling pathway of staphylococci internalization involves focal adhesion kinases (FAKs) and activated Src (Fowler et al., 2003; Agerer et al., 2005) that subsequently recruit cortactin to promote actin polymerization and mobilize the endocytic machinery (Figure 1B) (Agerer et al., 2005; Selbach and Backert, 2005). Downstream of the FAK-Src pathway, the activation of PI3K and Akt is also important for the internalization of S. aureus (Oviedo-Boyso et al., 2011; Wang et al., 2013), although the molecular pathway is still far from being fully understood. Previous studies have shown that internalization is inhibited by cytochalasin D (Ellington et al., 1999; Sinha et al., 1999) and is temperature dependent (Sinha et al., 1999, specifically, inhibited at 4 and $14^{\circ} \mathrm{C}$ and facilitated at $37^{\circ} \mathrm{C}$ versus room temperature). These results respectively show that the biological prerequisites for internalization are (1) a dynamic actin cytoskeleton and (2) a fluid host cell membrane. Since bacterial uptake can occur with heat-killed or fixed bacteria, this mechanism appears to be an active process on the cellular side only. However, it has also been shown that $S$. aureus can stimulate its own uptake by upregulating $\beta 1$ integrin expression in the host cell by secreting $\alpha$-hemolysin. In this case, the bacteria must be viable as the process requires both cellular and bacterial activity (Abel et al., 2011; Goldmann et al., 2016).

In summary, $S$. aureus adhere to cells via interactions between FnBPs, Fn, and $\alpha 5 \beta 1$ integrins. The resulting clustering of integrins may then be sufficient to trigger the signaling cascade involving FAK, Src, PI3K, and Akt. Finally, S. aureus mobilizes the actin cytoskeleton and possibly the endocytosis machinery to enter host cells (Figure 1).

The FnBP-Fn- $\alpha 5 \beta 1$ integrin pathway is widely acknowledged to be the main internalization process. However, other factors have been shown to affect the efficiency of this internalization. In epithelial cells indeed, the internalization efficiency is maximal when the bacterial FnBPs interact directly with Hsp60 at the cell surface (Dziewanowska et al., 2000). Whether Hsp60 acts as 
a co-receptor to strengthen the binding of FnBPs-Fn to $\alpha 5 \beta 1$ integrin or is involved in signal transduction has still to be investigated (Figure 1C). Another clue that the FnBP-Fn- $\alpha 5 \beta 1$ integrin pathway is not the only internalization mechanism is that blocking $\alpha 5 \beta 1$ integrin or the binding of Fn by FnBPs with an antibody does not fully prevent internalization by MG63 osteoblast cells (Testoni et al., 2011) and by primary keratinocytes (Kintarak et al., 2004). The latter even internalize the FnBP-defective strain DU5883. Together, these results suggest that alternative mechanisms are involved in the adhesion and internalization of $S$. aureus (Table 1).

\section{Secondary Mechanisms}

These mechanisms mainly involve bacterial serine aspartate repeat-containing protein $\mathrm{D}(\mathbf{S d r D})$, clumping factor A (ClfA), autolysin (Atl), and serine-rich adhesin for platelets (SraP). These proteins are MSCRAMMs and (except for Atl) have the cell-wall anchoring sequence LPXTG located in their C-terminal portion. Their N-terminal part contains a signal peptide for their secretion, followed by a ligand binding region mainly consisting of repeated sequences often rich in Serine. The mechanisms involving these proteins are Fn-independent: SdrD binds directly to Desmoglein 1 on the cell surface of keratinocytes and desquamated nasal cells (Corrigan et al., 2009; Askarian et al., 2016); ClfA interacts directly with host cells or through fibrinogen bridges (McDonnell et al., 2016; Claes et al., 2017); Atl seems to mediate $S$. aureus internalization via direct interactions with Hsc70 (Hirschhausen et al., 2010); and SraP adheres to A549 cells through the salivary scavenger protein gp340 (Yang et al., 2014) (Figure 1C). Note that the SdrD and Atl mechanisms require both bacterial and cellular activity as their efficiency depends on the expression of the bacterial and of the cellular interactor. Indeed, SdrD expression is upregulated following contact with neutrophils (Sitkiewicz et al., 2011) and Hsc70 production is stimulated by $S$. aureus infection of EA.hy 926 cells (Hirschhausen et al., 2010). Moreover, Atl has also been shown to be involved in the secretion of several S. aureus proteins, among which SdrD (Pasztor et al., 2010). Atl could thus have an "active" role as a secondary mechanism of internalization in some cells and act as a regulator of those secondary mechanisms in other cell types. More generally, these observations might reflect the fact that the bacteria can adapt their internalization strategy to the environmental conditions, i.e., an absence or scarcity of Fn, by finding alternative binding partners and/or by upregulating one side or the other of the adhesion machinery (e.g., SdrD and Atl). For instance, ClfA binds to annexin 2 on the surface of bovine mammary epithelial cells (MAC-T cells) (Ashraf et al., 2017) but has two receptors on the surface of endothelial cells, namely $\alpha v \beta 3$

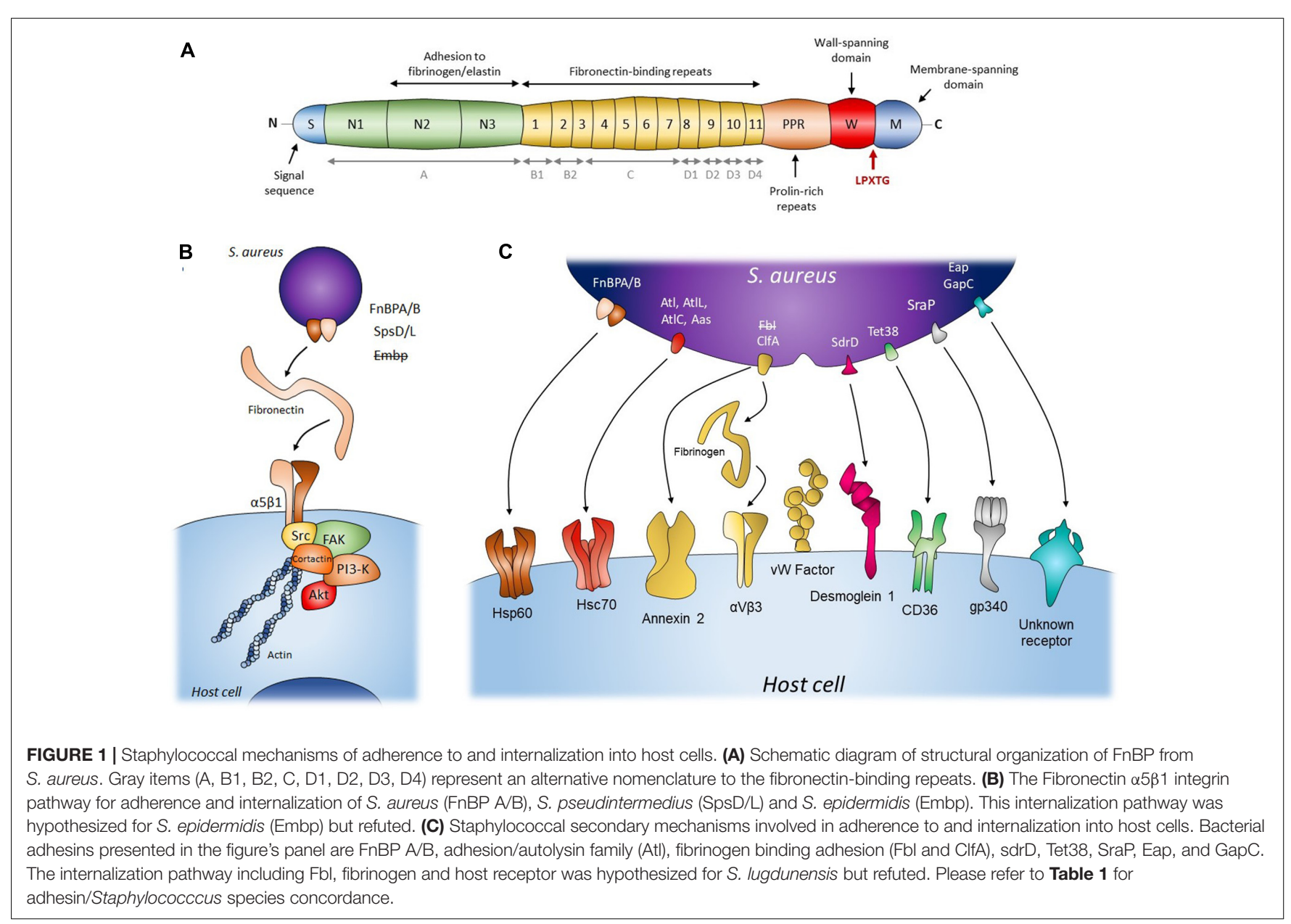


integrin using fibrinogen as a bridge (McDonnell et al., 2016) and von Willebrand factor using self-secreted von Willebrand factor binding protein as a bridge (Table $\mathbf{1}$ and Figure 1C) (Claes et al., 2017). This might increase its capacity to adhere to and possibly enter endothelial cells, thereby supporting its role in the pathogenesis of endocarditis. Fibrinogen binding also primes platelets aggregation and abscess formation and can lead to thromboembolic lesions in the heart during sepsis (McAdow et al., 2011; Malachowa et al., 2016).

In other cases, the alternative mechanisms may support the FnBP-Fn- $\alpha 5 \beta 1$ integrin-mediated uptake of $S$. aureus instead. This is notably illustrated by $S$. aureus extracellular adherence protein (Eap) that plays a role in the adherence to fibroblasts and epithelial cells independently of any binding to Fn or fibrinogen (Figure 1C) (Hussain et al., 2002). Recently, its deletion has been shown not to affect the adhesion and internalization steps, contradicting the early idea that its role was to compensate for FnBP function loss (Palma et al., 1999; Grundmeier et al., 2004). Rather, Eap seems to promote the adhesion and internalization of $S$. aureus and other pathogenic bacteria encountered in the context of polymicrobial skin infection (Bur et al., 2013). As for all the previously cited mechanisms, the molecular and signaling pathways underlying this activity are not understood yet and more work is needed to clarify whether Eap (1) is secreted and binds to $\alpha 5 \beta 1$ integrin via Fn, triggering the internalization cascade pathway and enhancing the uptake of bacteria bound to molecules other than $\alpha 5 \beta 1$ integrin in cells that express this integrin poorly; or (2) triggers actin-dependent phagocytosis, as its effect is fully blocked by cytochalasin D.

The need to elucidate the molecular and signaling pathways of these alternative mechanisms is highlighted by their phenotypical relevance. ClfA and SraP are involved in the pathogenesis of endocarditis. SdrD-mediated binding appears to help the bacteria survive in vivo (Askarian et al., 2017) - whether this is because of an increased ability to kill neutrophils and/or to invade cells is an open question. Also, unraveling whether internalization occurs via the same FAK/Src/PI3K/Akt cascade as for the FnBP-Fn- $\beta 1$ integrin pathway is essential as this may offer a potential avenue to treat chronic infections.

The minor mechanism involving GapC during the adhesion to and invasion of MAC-T cells is also noteworthy as it has been involved in the development of mastitis (Kerro-Dego et al., 2012). Finally, another point to bear in mind is that in addition to adhesins, other (probably less expected) molecules are involved in

TABLE 1 | Staphylococcal mechanisms of adherence to and internalization into host cells regarding of MSCRAMMs.

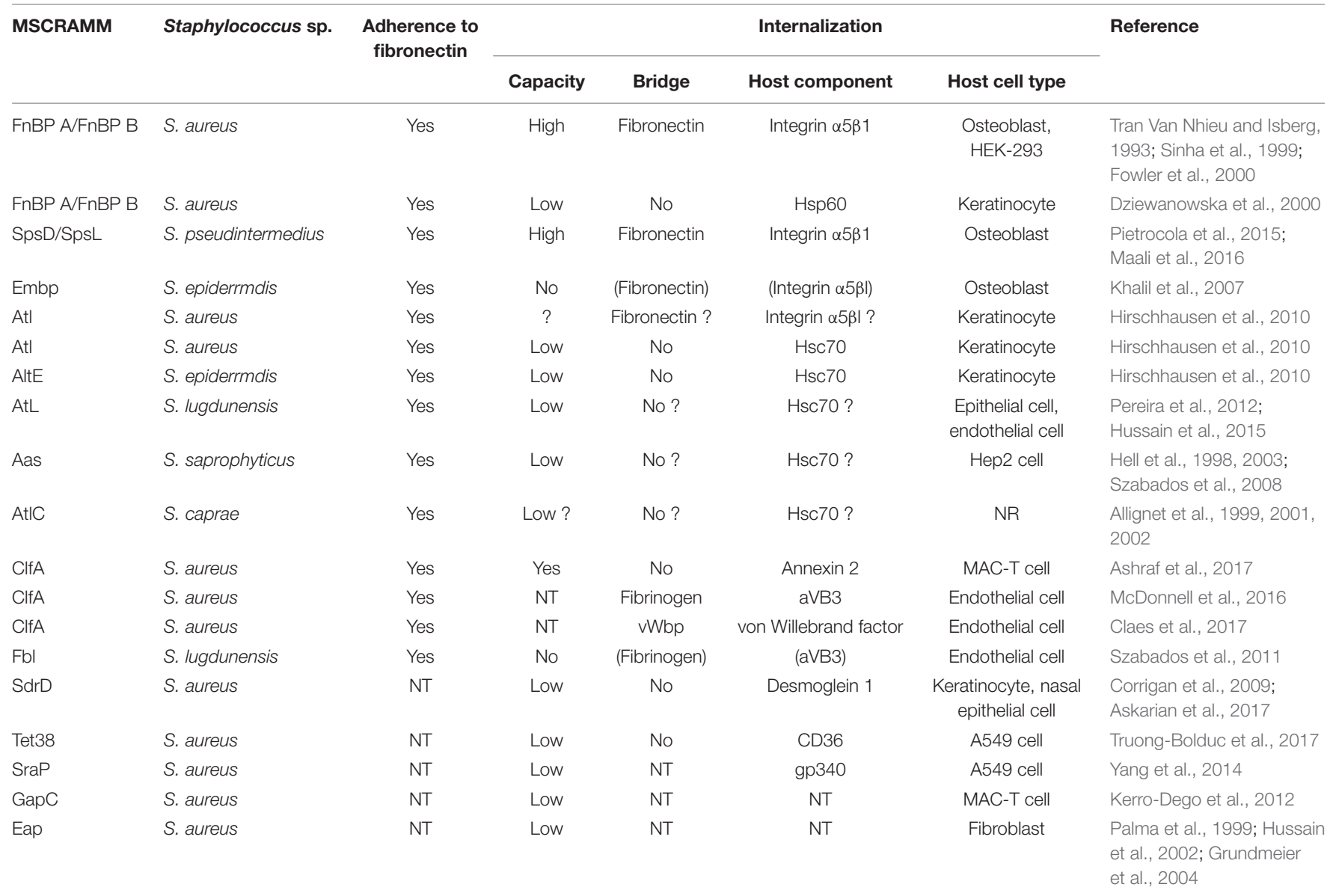

NR means not relevant. NT means not tested and not suggested in the literature or in this review. Question mark means that the internalization mechanism was suggested in the review or by other authors but not tested. () means that the hypothesized pathway was tested but refued. 
the adhesion to and invasion of NPPCs by S. aureus. In particular, the Tet38 efflux pump extrudes tetracycline and unsaturated free fatty acids but also interacts with CD36, a cellular transporter of long chain fatty acids, to trigger $S$. aureus adhesion and entry into A549 cells (Truong-Bolduc et al., 2017). Tet38 is also involved in phagosomal escape, facilitating the replication and persistence of S. aureus.

\section{Staphylococcus pseudintermedius}

Staphylococcus pseudintermedius is a coagulase-positive species mostly responsible for infections in dogs, notably necrotic skin lesions and bone and joint infections (Nazarali et al., 2015). However, human infections with $S$. pseudintermedius have also been described, revealing a risk of zoonotic transmission (Darlow et al., 2017; Lozano et al., 2017). Recently, Maali et al. (2016) performed a comparative study examining various CoNS species and reported that $S$. pseudintermedius was the only species that significantly adheres to human Fn and invades MG-63 cells with higher rates than S. aureus. This internalization does not occur in $\beta 1$ integrin-deficient murine osteoblasts and is mediated through SpsD and SpsL, two cell wall-anchored proteins that share homologies with $S$. aureus FnBPA and FnBPB (Pietrocola et al., 2015; Maali et al., 2016).

\section{Staphylococcus epidermidis AND OTHER COAGULASE-NEGATIVE STAPHYLOCOCCI}

The ability of S. epidermidis and other CoNS to bind fibronectin and to be internalized has also been investigated. The results of some of these studies and their interpretation are controversial however.

The first study of Fn binding in S. epidermidis and other CoNS species found that S. epidermidis was a good Fn binder (Switalski et al., 1983). However, this study also revealed huge variations in binding activity between $S$. epidermidis strains and between CoNS species. Later, Minhas et al. (1995) reported the presence of FnBP genes in S. epidermidis and other CoNS using PCR inside a repeat unit region (D1-D4) found in both $f n b A$ and $f n b B$ from $S$. aureus (Figure 1A). Still, no FnBP-like protein has clearly been identified in $S$. epidermidis so far and no other study has found FnBPs at genomic, transcriptomic, or protein levels in CoNS. In S. epidermidis, the giant extracellular matrix binding protein (Embp) has been shown to bind Fn (Williams et al., 2002). Embp harbors 59 "Found In Various Architectures" (FIVAR) domains, involved in Fn binding (Christner et al., 2010), and 38 protein G-related albumin-binding (GA). A recombinant protein containing the Fn-binding domain of Embp blocks $S$. epidermidis binding to Fn but not that of $S$. aureus. Conversely, the competitive use of recombinant FnBPB (D1-D4 units) has been shown to block the binding of $S$. aureus, but not of S. epidermidis, to Fn. S. epidermidis Embp and S. aureus FnBPs must therefore have distinct Fn interaction sites (Williams et al., 2002).
The internalization of S. epidermidis (and that of other CoNS) by NPPCs is a more controversial issue. While several studies have reported that $S$. epidermidis is internalized by different types of NPPCs, namely endothelial cells (Oviedo-Boyso et al., 2009), MAC-T cells (Almeida and Oliver, 2001) and human osteoblastlike MG-63 cells (Khalil et al., 2007), others have minimized its ability to invade MG-63 cells (Valour et al., 2013; Campoccia et al., 2016; Maali et al., 2016). Indeed, using appropriate infection conditions, Valour et al. (2013) and Campoccia et al. (2016) found that even with a multiplicity of infection (MOI) exceeding 500:1, the rate of $S$. epidermidis internalization $2 \mathrm{~h}$ after infection was very low. (The rate of $S$. epidermidis internalization at an MOI 500:1 was about 100 times lower than that of $S$. aureus at an MOI of 100:1.) Although the significance of such a low level of invasion by $S$. epidermidis remains unclear, the uptake can happen and the following mechanisms have been proposed and identified to explain this.

One tempting hypothesis is that the internalization of $S$. epidermidis by NPPCs occurs through a tripartite Embp-Fn$\alpha 5 \beta 1$ system analogous to the FnBP-Fn- $\alpha 5 \beta 1$ integrin process for $S$. aureus. Using a recombinant protein that blocks the Fn-binding domain of S. aureus FnBP, Khalil et al. (2007) completely inhibited the internalization of $S$. aureus but failed to block that of $S$. epidermidis. This fits with there being no FnBP in $S$. epidermidis, as discussed below, but does not refute the "Embp/Fn/ $\alpha 5 \beta 1$ integrin" hypothesis. However, the use of an anti- $\alpha 5 \beta 1$ integrin antibody was found to block the internalization of $S$. aureus but not that of $S$. epidermidis by MG-63 cells (Khalil et al., 2007). The uptake of S. epidermidis is therefore independent of the $\alpha 5 \beta 1$ integrin, which rules out the "Embp-Fn- $\alpha 5 \beta 1$ integrin" system and supports the intervention of secondary mechanisms.

In CoNS, the only internalization mechanism that has been described to date involves Atl. As mentioned above for Atl in $S$. aureus, $S$. epidermidis is internalized through direct interactions between AtlE and Hsc70 (Hirschhausen et al., 2010). This would seem to be the logical alternative mechanism for internalization as the other CoNS also have autolysins (Albrecht et al., 2012). However, existing studies only describe an ability to bind to Fn through autolysin and/or become internalized but do not offer a mechanistic explanation (Table 1) (Hell et al., 1998, 2003; Allignet et al., 1999, 2001, 2002; Szabados et al., 2008; Pereira et al., 2012; Hussain et al., 2015). It is also noteworthy that the involvement in the internalization process of the fibrinogenbinding protein $\mathrm{Fbl}$ produced in S. lugdunensis, a homolog of $S$. aureus ClfA, has also been investigated, with negative results (Szabados et al., 2011).

\section{CONCLUSION}

As this short review shows, adhesion to fibronectin is a major explanation for the virulence of staphylococci. In $S$. aureus and S. pseudintermedius, adhesion involves fibronectin and $\alpha 5 \beta 1$ integrin and leads to internalization in host cells, which favors intracellular persistence and chronic infections. There are alternative mechanisms through which staphylococci become 
internalized and these may explain why in the absence of a major Fn-related internalization mechanism, S. epidermidis still invades cells, albeit at a low level. It is not yet clear whether these secondary mechanisms are completely independent of the FnBP-Fn- $\alpha 5 \beta 1$ integrin pathway (i.e., substitute it) or if they support it by strengthening binding or by triggering internalization more effectively. Identifying (1) the detailed molecular mechanisms of the FnBP-Fn- $\alpha 5 \beta 1$ pathway, (2) the surface proteins involved in alternative mechanisms, and (3)

\section{REFERENCES}

Abel, J., Goldmann, O., Ziegler, C., Höltje, C., Smeltzer, M. S., Cheung, A. L., et al. (2011). Staphylococcus aureus evades the extracellular antimicrobial activity of mast cells by promoting its own uptake. J. Innate Immun. 3, 495-507. doi: $10.1159 / 000327714$

Agerer, F., Lux, S., Michel, A., Rohde, M., Ohlsen, K., and Hauck, C. R. (2005). Cellular invasion by Staphylococcus aureus reveals a functional link between focal adhesion kinase and cortactin in integrin-mediated internalisation. J. Cell Sci. 118(Pt 10), 2189-2200. doi: 10.1242/jcs.02328

Albrecht, T., Raue, S., Rosenstein, R., Nieselt, K., and Götz, F. (2012). Phylogeny of the staphylococcal major autolysin and its use in genus and species typing. J. Bacteriol. 194, 2630-2636. doi: 10.1128/JB.06609-11

Allignet, J., Aubert, S., Dyke, K. G., and El Solh, N. (2001). Staphylococcus caprae strains carry determinants known to be involved in pathogenicity: a gene encoding an autolysin-binding fibronectin and the ica operon involved in biofilm formation. Infect. Immun. 69, 712-718. doi: 10.1128/IAI.69.2.712-718. 2001

Allignet, J., England, P., Old, I., and El Solh, N. (2002). Several regions of the repeat domain of the Staphylococcus caprae autolysin, Atlc, are involved in fibronectin binding. FEMS Microbiol. Lett. 213, 193-197. doi: 10.1111/j.1574-6968.2002. tb11305.x

Allignet, J., Galdbart, J. O., Morvan, A., Dyke, K. G., Vaudaux, P., Aubert, S., et al. (1999). Tracking adhesion factors in Staphylococcus caprae strains responsible for human bone infections following implantation of orthopaedic material. Microbiology 145(Pt 8), 2033-2042. doi: 10.1099/13500872-145-8-2033

Almeida, R. A., and Oliver, S. P. (2001). Interaction of coagulase-negative Staphylococcus species with bovine mammary epithelial cells. Microb. Pathog. 31, 205-212. doi: 10.1006/mpat.2001.0465

Aly, R., Shinefield, H. R., Litz, C., and Maibach, H. I. (1980). Role of teichoic acid in the binding of Staphylococcus aureus to nasal epithelial cells. J. Infect. Dis. 141, 463-465. doi: 10.1093/infdis/141.4.463

Ashraf, S., Cheng, J., and Zhao, X. (2017). Clumping factor A of Staphylococcus aureus interacts with annexinA2 on mammary epithelial cells. Sci. Rep. 7:40608. doi: $10.1038 /$ srep40608

Askarian, F., Ajayi, C., Hanssen, A., van Sorge, N. M., Pettersen, I., Diep, D. B., et al. (2016). The interaction between Staphylococcus aureus sdrD and desmoglein 1 is important for adhesion to host cells. Sci. Rep. 6:22134. doi: 10.1038/srep22134

Askarian, F., Uchiyama, S., Valderrama, J. A., Ajayi, C., Sollid, J. U. E., van Sorge, N. M., et al. (2017). Serine-aspartate repeat protein D increases Staphylococcus aureus virulence and survival in blood. Infect. Immun. 85:e00559-16. doi: 10.1128/IAI.00559-16

Becker, K., Heilmann, C., and Peters, G. (2014). Coagulase-negative staphylococci. Clin. Microbiol. Rev. 27, 870-926. doi: 10.1128/CMR.00109-13

Bingham, R. J., Rudiño-Piñera, E., Meenan, N. A. G., Schwarz-Linek, U. Turkenburg, J. P., Höök, M., et al. (2008). Crystal structures of fibronectinbinding sites from Staphylococcus aureus FnBPA in complex with fibronectin domains. Proc. Natl. Acad. Sci. U.S.A. 105, 12254-12258. doi: 10.1073/pnas. 0803556105

Bur, S., Preissner, K. T., Herrmann, M., and Bischoff, M. (2013). The Staphylococcus aureus extracellular adherence protein promotes bacterial internalization by keratinocytes independent of fibronectin-binding proteins. J. Invest. Dermatol. 133, 2004-2012. doi: 10.1038/jid.2013.87

Burke, F. M., Di Poto, A., Speziale, P., and Foster, T. J. (2011). The A domain of fibronectin-binding protein B of Staphylococcus aureus contains a novel the role of these mechanisms, would be major steps toward more efficient anti-bacterial treatments for chronic staphylococci infections.

\section{AUTHOR CONTRIBUTIONS}

JJ and AD prepared the draft of the papers with the help of FL. FL revised the version of the text.

fibronectin binding site. FEBS J. 278, 2359-2371. doi: 10.1111/j.1742-4658.2011. 08159.x

Burke, F. M., McCormack, N., Rindi, S., Speziale, P., and Foster, T. J. (2010). Fibronectin-binding protein B variation in Staphylococcus aureus. BMC Microbiol. 10:160. doi: 10.1186/1471-2180-10-160

Campoccia, D., Testoni, F., Ravaioli, S., Cangini, I., Maso, A., Speziale, P., et al. (2016). Orthopedic implant infections: incompetence of Staphylococcus epidermidis, Staphylococcus lugdunensis, and Enterococcus faecalis to invade osteoblasts. J. Biomed. Mater. Res. A 104, 788-801. doi: 10.1002/jbm.a.35564

Christner, M., Franke, G. C., Schommer, N. N., Wendt, U., Wegert, K., Pehle, P., et al. (2010). The giant extracellular matrix-binding protein of Staphylococcus epidermidis mediates biofilm accumulation and attachment to fibronectin. Mol. Microbiol. 75, 187-207. doi: 10.1111/j.1365-2958.2009.06981.x

Claes, J., Liesenborghs, L., Peetermans, M., Veloso, T. R., Missiakas, D., Schneewind, O., et al. (2017). Clumping factor a, von Willebrand factor-binding protein and von Willebrand factor anchor Staphylococcus aureus to the vessel wall. J. Thromb. Haemost. 15, 1009-1019. doi: 10.1111/jth.13653

Corrigan, R. M., Miajlovic, H., and Foster, T. J. (2009). Surface proteins that promote adherence of Staphylococcus aureus to human desquamated nasal epithelial cells. BMC Microbiol. 9:22. doi: 10.1186/1471-2180-9-22

Darlow, C. A., Paidakakos, N., Sikander, M., and Atkins, B. (2017). A spinal infection with Staphylococcus pseudintermedius. BMJ Case Rep. 2017:bcr-2017221260. doi: 10.1136/bcr-2017-221260

Dziewanowska, K., Carson, A. R., Patti, J. M., Deobald, C. F., Bayles, K. W., and Bohach, G. A. (2000). Staphylococcal fibronectin binding protein interacts with heat shock protein 60 and integrins: role in internalization by epithelial cells. Infect. Immun. 68, 6321-6328. doi: 10.1128/IAI.68.11.6321-6328.2000

Edwards, A. M., Potter, U., Meenan, N. A. G., Potts, J. R., and Massey, R. C. (2011). Staphylococcus aureus keratinocyte invasion is dependent upon multiple high-affinity fibronectin-binding repeats within FnBPA. PLOS ONE 6:e18899. doi: 10.1371/journal.pone.0018899

Edwards, A. M., Potts, J. R., Josefsson, E., and Massey, R. C. (2010). Staphylococcus aureus host cell invasion and virulence in sepsis is facilitated by the multiple repeats within FnBPA. PLOS Pathog. 6:e1000964. doi: 10.1371/journal.ppat. 1000964

Eichenberger, E. M., Thaden, J. T., Sharma-Kuinkel, B., Park, L. P., Rude, T. H., Ruffin, F., et al. (2015). Polymorphisms in fibronectin binding proteins A and B among Staphylococcus aureus bloodstream isolates are not associated with arthroplasty infection. PLOS ONE 10:e0141436. doi: 10.1371/journal.pone. 0141436

Ellington, J. K., Reilly, S. S., Ramp, W. K., Smeltzer, M. S., Kellam, J. F., and Hudson, M. C. (1999). Mechanisms of Staphylococcus aureus invasion of cultured osteoblasts. Microb. Pathog. 26, 317-323. doi: 10.1006/mpat.1999.0272

Foster, T. J., Geoghegan, J. A., Ganesh, V. K., and Höök, M. (2014). Adhesion, invasion and evasion: the many functions of the surface proteins of Staphylococcus aureus. Nat. Rev. Microbiol. 12, 49-62. doi: 10.1038/ nrmicro3161

Fowler, T., Johansson, S., Wary, K. K., and Höök, M. (2003). Src kinase has a central role in in vitro cellular internalization of Staphylococcus aureus. Cell. Microbiol. 5, 417-426. doi: 10.1046/j.1462-5822.2003.00290.x

Fowler, T., Wann, E. R., Joh, D., Johansson, S., Foster, T. J., and Höök, M. (2000). Cellular invasion by Staphylococcus aureus involves a fibronectin bridge between the bacterial fibronectin-binding MSCRAMMS and host cell betal integrins. Eur. J. Cell Biol. 79, 672-679. doi: 10.1078/0171-933500104 
Goldmann, O., Tuchscherr, L., Rohde, M., and Medina, E. (2016). $\alpha$-hemolysin enhances Staphylococcus aureus internalization and survival within mast cells by modulating the expression of $\beta 1$ integrin. Cell. Microbiol. 18, 807-819. doi: $10.1111 / \mathrm{cmi} .12550$

Grundmeier, M., Hussain, M., Becker, P., Heilmann, C., Peters, G., and Sinha, B. (2004). Truncation of fibronectin-binding proteins in Staphylococcus aureus strain Newman leads to deficient adherence and host cell invasion due to loss of the cell wall anchor function. Infect. Immun. 72, 7155-7163. doi: 10.1128/IAI. 72.12.7155-7163.2004

Hell, W., Meyer, H. G., and Gatermann, S. G. (1998). Cloning of aas, a gene encoding a Staphylococcus saprophyticus surface protein with adhesive and autolytic properties. Mol. Microbiol. 29, 871-881. doi: 10.1046/j.1365-2958. 1998.00983.x

Hell, W., Reichl, S., Anders, A., and Gatermann, S. (2003). The autolytic activity of the recombinant amidase of Staphylococcus saprophyticus is inhibited by its own recombinant GW repeats. FEMS Microbiol. Lett. 227, 47-51. doi: 10.1016/ S0378-1097(03)00647-5

Hirschhausen, N., Schlesier, T., Schmidt, M. A., Götz, F., Peters, G., and Heilmann, C. (2010). A novel staphylococcal internalization mechanism involves the major autolysin Atl and heat shock cognate protein Hsc70 as host cell receptor. Cell. Microbiol. 12, 1746-1764. doi: 10.1111/j.1462-5822.2010. 01506.x

Hos, N. J., Rieg, S., Kern, W. K., Jonas, D., Fowler, V. G., Higgins, P. G., et al. (2015). Amino acid alterations in fibronectin binding protein a (FnBPA) and bacterial genotype are associated with cardiac device related infection in Staphylococcus aureus bacteraemia. J. Infect. 70, 153-159. doi: 10.1016/j.jinf.2014.09.005

Hussain, M., Haggar, A., Heilmann, C., Peters, G., Flock, J. I., and Herrmann, M. (2002). Insertional inactivation of Eap in Staphylococcus aureus strain Newman confers reduced staphylococcal binding to fibroblasts. Infect. Immun. 70, 2933-2940. doi: 10.1128/IAI.70.6.2933-2940.2002

Hussain, M., Steinbacher, T., Peters, G., Heilmann, C., and Becker, K. (2015). The adhesive properties of the Staphylococcus lugdunensis multifunctional autolysin AtlL and its role in biofilm formation and internalization. Int. J. Med. Microbiol. 305, 129-139. doi: 10.1016/j.ijmm.2014.11.010

Jönsson, K., Signäs, C., Müller, H. P., and Lindberg, M. (1991). Two different genes encode fibronectin binding proteins in Staphylococcus aureus. The complete nucleotide sequence and characterization of the second gene. Eur. J. Biochem. 202, 1041-1048. doi: 10.1111/j.1432-1033.1991.tb16468.x

Kerro Dego, O., van Dijk, J. E., and Nederbragt, H. (2002). Factors involved in the early pathogenesis of bovine Staphylococcus aureus mastitis with emphasis on bacterial adhesion and invasion. A review. Vet. Q. 24, 181-198. doi: 10.1080/ 01652176.2002 .9695135

Kerro-Dego, O., Prysliak, T., Perez-Casal, J., and Potter, A. A. (2012). Role of GapC in the pathogenesis of Staphylococcus aureus. Vet. Microbiol. 156, 443-447. doi: $10.1016 /$ j.vetmic.2011.11.018

Khalil, H., Williams, R. J., Stenbeck, G., Henderson, B., Meghii, S., and Nair, S. P. (2007). Invasion of bone cells by Staphylococcus epidermidis. Microbes Infect. 9, 460-465. doi: 10.1016/j.micinf.2007.01.002

Kintarak, S., Whawell, S. A., Speight, P. M., Packer, S., and Nair, S. P. (2004). Internalization of Staphylococcus aureus by human keratinocytes. Infect. Immun. 72, 5668-5675. doi: 10.1128/IAI.72.10.5668-5675.2004

Löffler, B., Tuchscherr, L., Niemann, S., and Peters, G. (2014). Staphylococcus aureus persistence in non-professional phagocytes. Int. J. Med. Microbiol. 304, 170-176. doi: 10.1016/j.ijmm.2013.11.011

Lowy, F. D. (1998). Staphylococcus aureus infections. N. Engl. J. Med. 339, 520-532. doi: 10.1056/NEJM199808203390806

Lozano, C., Rezusta, A., Ferrer, I., Pérez-Laguna, V., Zarazaga, M., Ruiz-Ripa, L., et al. (2017). Staphylococcus pseudintermedius human infection cases in Spain: dog-to-human transmission. Vector Borne Zoonotic Dis. 17, 268-270. doi: $10.1089 / \mathrm{vbz} .2016 .2048$

Maali, Y., Martins-Simões, P., Valour, F., Bouvard, D., Rasigade, J. P., Bes, M., et al. (2016). Pathophysiological mechanisms of Staphylococcus non-aureus bone and joint infection: interspecies homogeneity and specific behavior of S. pseudintermedius. Front. Microbiol. 7:1063. doi: 10.3389/fmicb.2016.01063

Malachowa, N., Kobayashi, S. D., Porter, A. D., Braughton, K. R., Scott, D. P., Gardner, D. J., et al. (2016). Contribution of Staphylococcus aureus coagulases and clumping factor A to abscess formation in a rabbit model of skin and soft tissue infection. PLOS ONE 11:e0158293. doi: 10.1371/journal.pone.0158293
McAdow, M., Kim, H. K., Dedent, A. C., Hendrickx, A. P. A., Schneewind, O., and Missiakas, D. M. (2011). Preventing Staphylococcus aureus sepsis through the inhibition of its agglutination in blood. PLOS Pathog. 7:e1002307. doi: 10.1371/journal.ppat.1002307

McDonnell, C. J., Garciarena, C. D., Watkin, R. L., McHale, T. M., McLoughlin, A., Claes, J., et al. (2016). Inhibition of major integrin $\alpha \mathrm{v} \beta 3$ reduces Staphylococcus aureus attachment to sheared human endothelial cells. J. Thromb. Haemost. 14, 2536-2547. doi: 10.1111/jth.13501

Minhas, T., Ludlam, H. A., Wilks, M., and Tabaqchali, M. (1995). Detection by PCR and analysis of the distribution of a fibronectin-binding protein gene (fbn) among staphylococcal isolates. J. Med. Microbiol. 42, 96-101. doi: 10.1099/ 00222615-42-2-96

Moormeier, D. E., and Bayles, K. W. (2017). Staphylococcus aureus biofilm: a complex developmental organism. Mol. Microbiol. 104, 365-376. doi: 10.1111/ mmi.13634

Nazarali, A., Singh, A., Moens, N. M. M., Gatineau, M., Sereda, C., Fowler, D., et al. (2015). Association between methicillin-resistant Staphylococcus pseudintermedius carriage and the development of surgical site infections following tibial plateau leveling osteotomy in dogs. J. Am. Vet. Med. Assoc. 247, 909-916. doi: 10.2460/javma.247.8.909

Oviedo-Boyso, J., Bravo-Patiño, A., Cajero-Juárez, M., Valdez-Alarcón, J. J., and Baizabal-Aguirre, V. M. (2009). TNF-alpha reduces the level of Staphylococcus epidermidis internalization by bovine endothelial cells. FEMS Microbiol. Lett. 292, 92-99. doi: 10.1111/j.1574-6968.2008.01469.x

Oviedo-Boyso, J., Cortés-Vieyra, R., Huante-Mendoza, A., Yu, H. B., ValdezAlarcón, J. J., Bravo-Patiño, A., et al. (2011). The hosphoinositide-3-Kinase-Akt signaling pathway is important for Staphylococcus aureus internalization by endothelial cells. Infect. Immun. 79, 4569-4577. doi: 10.1128/IAI.05303-11

Palma, M., Haggar, A., and Flock, J. I. (1999). Adherence of Staphylococcus aureus is enhanced by an endogenous secreted protein with broad binding activity. J. Bacteriol. 181, 2840-2845.

Pasztor, L., Ziebandt, A. K., Nega, M., Schlag, M., Haase, S., Franz-Wachtel, M., et al. (2010). Staphylococcal major autolysin (Atl) is involved in excretion of cytoplasmic proteins. J. Biol. Chem. 285, 36794-36803. doi: 10.1074/jbc.M110. 167312

Patti, J. M., Allen, B. L., McGavin, M. J., and Höök, M. (1994). MSCRAMMmediated adherence of microorganisms to host tissues. Annu. Rev. Microbiol. 48, 585-617. doi: 10.1146/annurev.mi.48.100194.003101

Peacock, S. J., Day, N. P., Thomas, M. G., Berendt, A. R., and Foster, T. J. (2000). Clinical isolates of Staphylococcus aureus exhibit diversity in fnb genes and adhesion to human fibronectin. J. Infect. 41, 23-31. doi: 10.1053/jinf.2000.0657

Pereira, E. M., Teixeira, C. A. A., Alvarenga, A. L. M., Schuenck, R. P., GiambiagiDemarval, R., Holandino, C., et al. (2012). A Brazilian lineage of Staphylococcus lugdunensis presenting rough colony morphology may adhere to and invade lung epithelial cells. J. Med. Microbiol. 61(Pt 4), 463-469. doi: 10.1099/jmm.0. 033001-0

Pietrocola, G., Gianotti, V., Richards, A., Nobile, G., Geoghegan, J. A., Rindi, S., et al. (2015). Fibronectin binding proteins SpsD and SpsL both support invasion of canine epithelial cells by Staphylococcus pseudintermedius. Infect. Immun. 83, 4093-4102. doi: 10.1128/IAI.00542-15

Roche, F. M., Downer, R., Keane, F., Speziale, P., Park, P. W., and Foster, T. J. (2004). The N-terminal A domain of fibronectin-binding proteins A and B promotes adhesion of Staphylococcus aureus to elastin. J. Biol. Chem. 279, 38433-38440. doi: 10.1074/jbc.M402122200

Schwarz-Linek, U., Werner, J. M., Pickford, A. R., Gurusiddappa, S., Kim, J. H., Pilka, E. S., et al. (2003). Pathogenic bacteria attach to human fibronectin through a tandem beta-zipper. Nature 423, 177-181. doi: 10.1038/nature01589

Selbach, M., and Backert, S. (2005). Cortactin: an achilles' heel of the actin cytoskeleton targeted by pathogens. Trends Microbiol. 13, 181-189. doi: 10.1016/j.tim.2005.02.007

Shinji, H., Yosizawa, Y., Tajima, A., Iwase, T., Sugimoto, S., Seki, K., et al. (2011). Role of fibronectin-binding proteins A and B in in vitro cellular infections and in vivo septic infections by Staphylococcus aureus. Infect. Immun. 79, 2215-2223. doi: 10.1128/IAI.00133-11

Sinha, B., François, P. P., Nüsse, O., Foti, M., Hartford, O. M., Vaudaux, P., et al. (1999). Fibronectin-binding protein acts as Staphylococcus aureus invasin via fibronectin bridging to integrin alpha5beta1. Cell. Microbiol. 1, 101-117. doi: 10.1046/j.1462-5822.1999.00011.x 
Sitkiewicz, I., Babiak, I., and Hryniewicz, W. (2011). Characterization of transcription within $S d r$ region of Staphylococcus aureus. Antonie Van Leeuwenhoek 99, 409-416. doi: 10.1007/s10482-010-9476-7

Switalski, L. M., Rydén, C., Rubin, K., Ljungh, A., Höök, M., and Wadström, T. (1983). Binding of fibronectin to Staphylococcus strains. Infect. Immun. 42, $628-633$.

Szabados, F., Kleine, B., Anders, A., Kaase, M., Sakinç, T., Schmitz, I., et al. (2008). Staphylococcus saprophyticus ATCC 15305 is internalized into human urinary bladder carcinoma cell line 5637. FEMS Microbiol. Lett. 285, 163-169. doi: 10.1111/j.1574-6968.2008.01218.x

Szabados, F., Marlinghaus, L., Korte, M., Neumann, S., Kaase, M., and Gatermann, S. G. (2011). Fbl is not involved in the invasion of eukaryotic epithelial and endothelial cells by Staphylococcus lugdunensis. FEMS Microbiol. Lett. 324, 48-55. doi: 10.1111/j.1574-6968.2011.02382.x

Testoni, F., Montanaro, L., Poggi, A., Visai, L., Campoccia, D., and Arciola, C. R. (2011). Internalization by osteoblasts of two Staphylococcus aureus clinical isolates differing in their adhesin gene pattern. Int. J. Artif. Organs 34, 789-798. doi: 10.5301/ijao.5000058

Tran Van Nhieu, G., and Isberg, R. R. (1993). Bacterial internalization mediated by beta 1 chain integrins is determined by ligand affinity and receptor density. EMBO J. 12, 1887-1895.

Truong-Bolduc, Q. C., Khan, N. S., Vyas, J. M., and Hooper, D. C. (2017). Tet38 efflux pump affects Staphylococcus aureus internalization by epithelial cells through interaction with CD36 and contributes to bacterial escape from acidic and nonacidic phagolysosomes. Infect. Immun. 85:e00862-16. doi: 10.1128/IAI. 00862-16

Valour, F., Trouillet-Assant, S., Rasigade, J. P., Lustig, S., Chanard, E., Meugnier, H., et al. (2013). Staphylococcus epidermidis in orthopedic device infections: the role of bacterial internalization in human osteoblasts and biofilm formation. PLOS ONE 8:e67240. doi: 10.1371/journal.pone.0067240

Wang, J. H., Zhang, K., Wang, N., Qiu, X. M., Wang, Y. B., and He, P. (2013) Involvement of phosphatidylinositol 3-kinase/Akt signaling pathway in $\beta 1$ integrin-mediated internalization of Staphylococcus aureus by alveolar epithelial cells. J. Microbiol. 51, 644-650. doi: 10.1007/s12275-013-3040-x

Wann, E. R., Gurusiddappa, S., and Hook, M. (2000). The fibronectin-binding MSCRAMM FnbpA of Staphylococcus aureus is a bifunctional protein that also binds to fibrinogen. J. Biol. Chem. 275, 13863-13871. doi: 10.1074/jbc.275.18. 13863

Williams, R. J., Henderson, B., Sharp, L. J., and Nair, S. P. (2002). Identification of a fibronectin-binding protein from Staphylococcus epidermidis. Infect. Immun. 70, 6805-6810. doi: 10.1128/IAI.70.12.6805-6810.2002

Yang, Y. H., Jiang, Y. L., Zhang, J., Wang, L., Bai, X. H., Zhang, S. J., et al. (2014). Structural insights into SraP-mediated Staphylococcus aureus adhesion to host cells. PLOS Pathog. 10:e1004169. doi: 10.1371/journal.ppat. 1004169

Conflict of Interest Statement: The authors declare that the research was conducted in the absence of any commercial or financial relationships that could be construed as a potential conflict of interest.

Copyright (c) 2017 Josse, Laurent and Diot. This is an open-access article distributed under the terms of the Creative Commons Attribution License (CC BY). The use, distribution or reproduction in other forums is permitted, provided the original author(s) or licensor are credited and that the original publication in this journal is cited, in accordance with accepted academic practice. No use, distribution or reproduction is permitted which does not comply with these terms. 